\title{
Medical students' awareness of radiation exposure related to radiological imaging procedures
}

\author{
Katarzyna Jończyk-Potoczna ${ }^{1}$, Lidia Strzelczuk-Judka ${ }^{1}$ Ewa Szłyk ${ }^{1,2}$, Łucja Stefaniak ${ }^{1,2}$ \\ ${ }^{1}$ Department of Pediatric Radiology, Poznan University of Medical Sciences, Poland \\ 2 Student Scientific Circle of Pediatric Radiology, Poznan University of Medical Sciences, Poland
}

\begin{abstract}
Introduction. Current advancements in fields of medical sciences resulted in an increase of imaging examinations with a use of ionising radiation. Such increase leads to justified concerns about its possible consequences.

Aim. Assessment of awareness and level of knowledge of medicine students in terms of ionising radiation Material and methods. Statistical analysis of 207 surveys of medical students from University of Medical Sciences in Poznan (UMP), Poland with use of Statistica software

Results. There was no significant difference between the results of the assessment related to gender, year of studies or attendance to radiology classes.

Conclusions. Students level of knowledge about the ionising radiation was unsatisfactory. A change in training program can be a benefit for patients and physicians.
\end{abstract}

Keywords: medical students, ionising radiation, radiological protection.

\section{Introduction}

The advancement and development of imaging procedures involving ionising radiation, growing availability of CT (Computed Tomography) procedures, angiography (DSA - Digital Subtraction Angiography), X-Ray tests resulted in an increase of imaging examinations in everyday clinical practice [1]. Such increase refers especially to CT examinations, the number of which significantly grew in last 10 years [2]. Despite of direct benefits for every patient due to the $\mathrm{CT}$, X-Ray or DSA procedures, unjustified use of these raises concerns and controversy about the possibly higher risk of subsequent neoplasms $[2,3]$, especially while exposure of the pediatric patients to the ionising radiation is concerned [3]. Patients who undergo series of examinations with the use of X-Ray as a standard part of procedures in various clinical units also belong to a higher risk group, in terms of induced neoplasms.
The influence of the radiation on the tissues leads to a change of their cell structure, particularly when the DNA chain sequence is damaged. It is closely related to the gene or chromosomal mutation, which might result in a multistage development of the neoplasm [4]. It has been proved that the radiation itself might cause a kind of genetic instability in cells, which enhances the risk and rapidity of mutations and other genetic changes after the multiple cellular reproductions. Further proofs indicate the fact that in a cytoplasm exposed to radiation the pace of mutations is significantly increased. Genetic aberrations, including induction in genome mutation and changes of gene expression might also occur in newly created cells that were not directly irradiated [5].

Effective dose characterises the degree of whole body exposure to radiation even upon irradiation of only certain parts of the body. The dose varies and 
depends on the examination technique (CT,CR - chest $X$-Rays, DSA), age of the patient and time of the exposure.

Carcinogenesis is one of the stochastic effects of the radiation with using $X$ - Rays, and appears with a specific probability, which means that it can, but it does not have to occur. There is no trigger dose of ionising radiation, at which the process of carcinogenesis happens. However, the higher is the exposure dose, the higher is also the probability of carcinogenesis, minding the fact that it may occur many years after exposure. It is also thought that the danger of ionising radiation at the early stages of life increases the risk of cancer $[2,4,6]$.

Justified concerns related to a high level of radiation exposure, especially increased lifelong risk of cancer encourage to seek solutions on an international stage. This problem was shown to the EURATOM community, which issued a set of recommendations, inter alia including aspects of radiological protection in training program at medical schools [7].

Until today many researches assessing knowledge of clinicians of different specializations about the ionising radiation and its risk of further consequences were conducted. However, the results were unsatisfactory [8-11].

It seems that the majority of physicians, despite completed training, does not have sufficient knowledge about the ionising radiation and doses acquired by the patients who undergo commonly made CT, DSA and X-Ray procedures.

Lack of knowledge about the extent of exposure of the whole organism to the ionizing radiation becomes significantly important, when the number of patients who undergo incorrect or repeated examinations is taken under consideration [8-11].

Although publications referring to the state of medical students' knowledge of radiology already exist [7], we decided that a specific area of awareness among the UMP students concerning doses of radiation related to different imaging examinations and assessing the risk of neoplasm development induced by the radiation requires detailed study.

\section{Aim}

The assessment of awareness and knowledge of the students related to exposure to radiation associated with imaging diagnostic procedures and radiation doses acquired by patients undergoing examinations with $a$ use of ionizing radiation.

\section{Material and methods}

Students participating in a Radiologic Scientific Club prepared a survey (Figure 1) which included one-choice questions about doses of radiation accompanying different radiological procedures. Participants were asked to fill the surveys anonymously and answer every question on their own. The survey consisted of two sections. The first section included basic information about the participants: year of medical studies, gender, attending radiology lecture. The second one was a set of 13 questions prepared to assess the level of both awareness and knowledge about the radiation. Correct answers in the sections were awarded by 1 point for each question, whereas incorrect - by 0 points. The maximum score was 13 points. All participants agreed to take part in a survey. The results were presented regarding gender, studying year, attending radiology lecture.

We compared the accuracy of the answers in the section 2 of the survey between the following research groups: men vs. women, 1 year of students vs. other years, 3 year of studies vs. other years, 4 year of studies vs. other years, 5 year of studies vs. other years, 6 year of studies vs. other years, students before radiology lectures vs. students after attending radiology lectures. We also analysed the correlation between the year of studies and number of correct answers in the test. We examined the correlation between: gender and accuracy of answer to each question, year of studies and accuracy of answer to each question, radiology lecture attendance and accuracy of answer to each question.

Statistical analysis was made with a use of Statistica software. In order to assess the normal distribution a Student's t- test for independent samples was used (assuming the value of $p$ lower than 0.05 as statistically significant). For the trials not showing a normal distribution a U Mann-Whitney test was used. Spearman's rank correlation coefficient was used for correlation tests.

\section{Results}

207 students from Poznan University of Medical Sciences took part in a survey research. The group consisted of 141 women and 66 men, radiology lecture attendance was declared by 110 participants, 97 students were before the radiology course. Among the students there were 18 from the first year, 21 from the second, 39 from the third, 69 from the fourth, 39 from the fifth, 21 from the sixth year. 
We kindly ask you to complete the following anonymous survey. The results shall verify the awareness of medical students regarding radiation doses associated with diagnostic imaging procedures and radiation protection. They will be the basis of a scientific study, so we count on your honesty. This is not a test for the mark! On behalf of the research team, thank you very much for your time.

1. Gender

$\mathrm{F}$

M

2. Field of study.
M.D. program
D.D.S program

3. Year of study.

I

II

III IV

$\mathrm{V}$

VI

4. Did you have classes in radiology?

Yes

No

5. Who bears higher risks associated with ionizing radiation procedures?
a child
an adult
it is the same in both

6. Who bears higher risks associated with being subjected to radiological examination of the chest?

female male it is the same in both

7. What $\%$ of diagnosed tumors per year may be associated with the CT study?
$0.1-0.2 \%$
$0.5 \%$
$1-2 \%$
around $5 \%$

8. What is the radiation dose associated with chest radiograph?
$0.01 \mathrm{mSv}$
$0.1 \mathrm{mSv}$
$1 \mathrm{mSv}$
$11 \mathrm{mSv}$
$20 \mathrm{mSv}$

9. For comparison, how many times is the radiation dose higher in the following studies compared to chest radiograph?

$\begin{array}{lllll}\text { Chest CT } & 7 \mathrm{x} & 50 \mathrm{x} & 70 \mathrm{x} & 100 \mathrm{x} \\ \text { Head CT } & 10 \mathrm{x} & 20 \mathrm{x} & 50 \mathrm{x} & 100 \mathrm{x} \\ \text { Abdomen CT } & 10 \mathrm{x} & 20 \mathrm{x} & 40 \mathrm{x} & 100 \mathrm{x} \\ \text { Mammography } & 1 \mathrm{x} & 7 \mathrm{x} & 20 \mathrm{x} & 50 \mathrm{x} \\ \text { Bone scintigraphy } & 20 \mathrm{x} & 40 \mathrm{x} & 80 \mathrm{x} & 100 \mathrm{x} \\ \text { Coronary angiography } & 50 \mathrm{x} & 80 \mathrm{x} & 100 \mathrm{x} & 200 \mathrm{x}\end{array}$

10. What is the dose limit to the whole body for the general Polish population (excluding people vocationally exposed to ionizing radiation)?
$1 \mathrm{mSv} / \mathrm{year}$
$5 \mathrm{mSv} /$ year
$10 \mathrm{mSv} /$ year
$20 \mathrm{mSv} /$ year
$50 \mathrm{mSv} / \mathrm{year}$

11. What is the dose limit for people vocationally exposed to ionizing radiation classified as $A$ category in Poland?
$1 \mathrm{mSv} / \mathrm{year}$
$5 \mathrm{mSv} / \mathrm{year}$
$10 \mathrm{mSv} /$ year
$20 \mathrm{mSv} / \mathrm{year}$
$50 \mathrm{mSv} / \mathrm{year}$

12. What is mean dose due to natural background radiation?
$0.3 \mathrm{mSv}$
$3 \mathrm{mSv}$
$30 \mathrm{mSv}$
$300 \mathrm{mSV}$

Figure 1. An anonymous survey assessing the awareness of medical students about doses of radiation related to imaging examinations and radiological protection

The results of statistical analysis were as followed: no significant statistical difference was found in the number of correct answers in terms of gender (p-0.23) (Figure 2), as well as attendance to radiology classes (p-0.14) (Figure 3). No strong difference was also found between the students from the first year of studies and older ones. There was also no correlation between the year of studies and the average number of points (Figure 4).

The least difficult question was this with a number 5 (93.7\% of correct answers) - majority of participants pointed to a higher radiation risk among children. Little more than a half of participants answered correctly to a question concerning a higher radiation risk in terms of gender of patient undergoing chest $X$-ray examination. In question number 7 percentage of correct answers was 25.6 . Only $35.7 \%$ of students indicated a dose of $0.1 \mathrm{mSv}$ acquired during a chest X-ray and because of that, question number 9 was also troublesome, especially a) point $-94.7 \%$ of participants underestimated an effective dose acquired during a chest computed tomography compared to chest X-Ray. Slightly better was the percentage of correct answers for question $9 b$, 9d and 9e - consequently 30, 38 and 29\% of correct answers. Moving onto questions $9 \mathrm{c}$ and $9 \mathrm{f}$ - related to abdomen computed tomography and coronary angiog- 


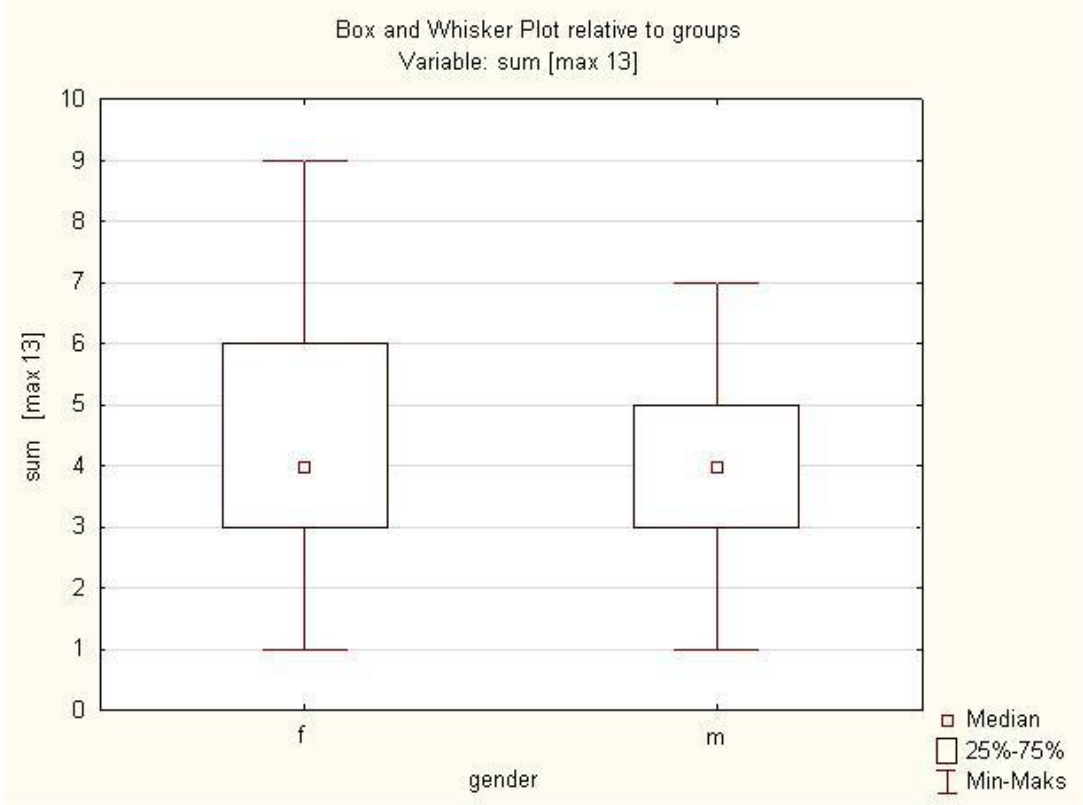

Figure 2. A graph of average number of points depending on gender, with a use of median percentile range (25-75) of minimum and maximum number of acquired points

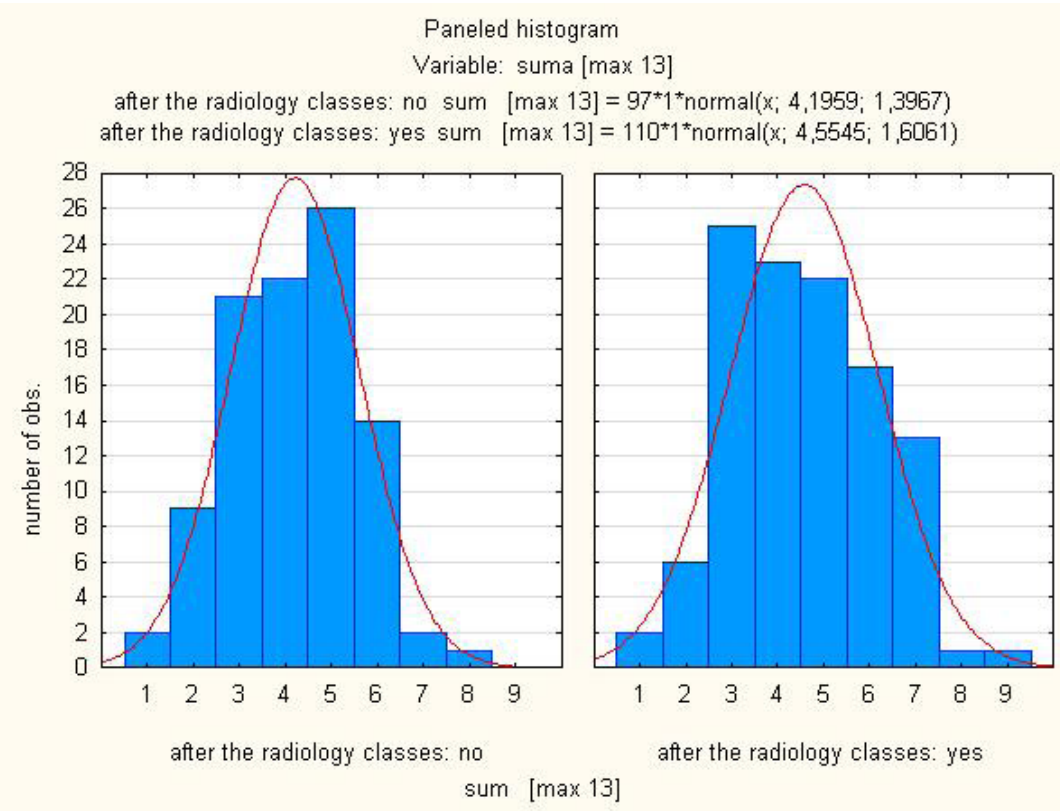

Figure 3. Visualisation of the amount of points obtained in the group before and after radiology and diagnostic imaging classes

raphy, respectively 18.8 and $11.6 \%$ of participants gave correct answers. Only $15 \%$ of answers where correct, when it comes to question about a trigger dose for total population in Poland, whereas question about such dose for people vocationally exposed to radiation was answered correctly by $27.5 \%$ of students. A value of an average natural background radiation was correctly stated by less than $40 \%$ of students.

\section{Discussion}

The use of ionizing radiation in imaging diagnostics requires experience and decent knowledge about the radiation dose related to all different radiological procedures.

In answer to increasing number of imaging examinations in everyday diagnostics particular impact on 
Without grouping

Year of studies: Least Mean Squares

Current efect: $F(5,201)=1,7538, p=, 12400$

Vertical bars represent confidence intervals 0.95

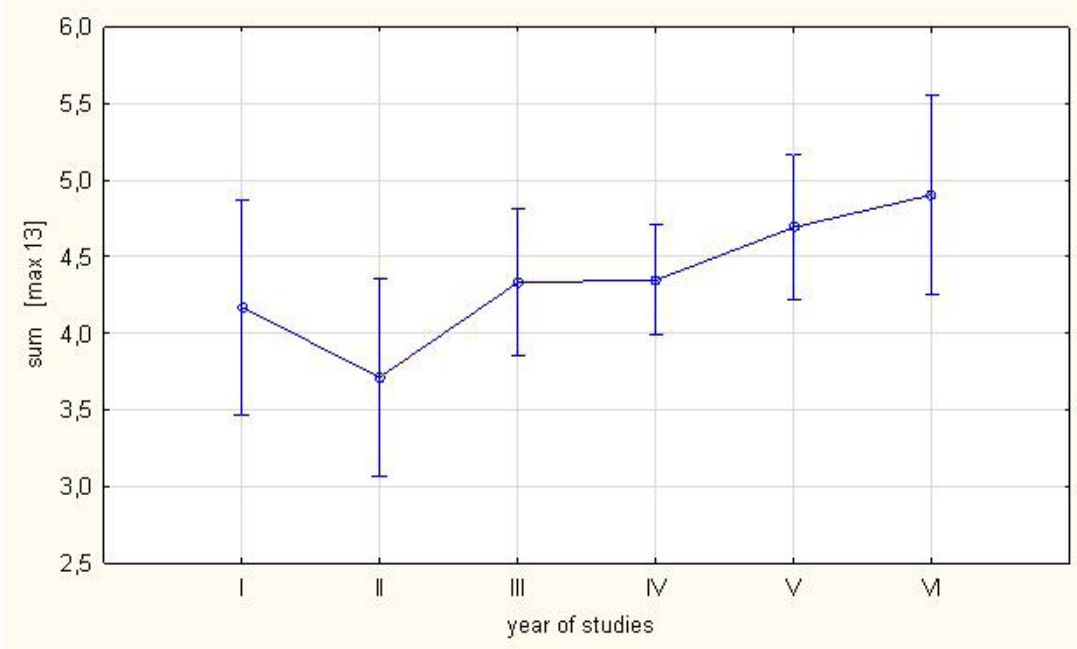

Figure 4. A graph of correlation between the year of studies and the average points scored

acquiring knowledge about the protection against radiation and its law regulations by students of medicine during basic radiology course seems to be reasonable. The level of knowledge expected from students of medicine should represent the current state of cognizance of lecturers. Still, multiple concerns about the knowledge of doctors in this topic already exist.

This gap in knowledge should be taken into consideration while preparing a curriculum for medical studies. Single researches stating a core curriculum for radiology studies has been already made [12]. By providing a clear, reachable curriculum, researches quoted before are a valuable source of information helping in developing education about the radiological protection [12]. The results of our study indicate the fact that the awareness about radiological protection among the medical students should be taught in the first place in order to improve the knowledge of future clinicians.

The fact, that no significant statistic difference in a level of knowledge of students in a group before and after radiology lecture attendance was detected might be related to the amount of material that must be learned by student during the course. According to international data $[7,13]$ an additional course in radiological protection and radiation risk significantly improves the knowledge of medical students in this topic. Increasing accessibility of medical information for patients and their caretakers as well as law regu- lations concerning professional liability should be an incentive for teaching students and physicians.

The survey, prepared by students of the fifth year participating in additional radiology courses as a part of Radiological Scientific Circle, was an outcome of a strong will to draw attention to problems due to the escalation in number of imaging examinations performed without proper understanding of the side effects of such procedures.

\section{Conclusions}

Our research has shown a poor knowledge of radiation risk among the students of medicine, regardless of gender, year of studies or an already finished course in radiology. Modifying the training program in terms of ionising radiation risk and choice of other rayless imaging techniques might be beneficial for both patients and future physicians.

\section{References}

1. Brenner $D$, et al. Estimated risks of radiation-induced fatal cancer from pediatric CT. AR Am J Roentgenol. 2001;176(2):289-96.

2. Pearce MS, et al. Radiation exposure from CT scans in childhood and subsequent risk of leukaemia and brain tumours: a retrospective cohort study. Lancet. 2012; 380:499-505.

3. Mathews JD, et al. Cancer risk in 680000 people exposed to computed tomography scans in childhood or 
adolescence: data linkage study of 11 million Australian. BJ. 2013;346:f2360.

4. Leenhouts HP, Chadwick KH. The molecular basis of stochastic and nonstochastic effects. Health Phys. 1989;57 (Suppl 1):343-8.

5. Little J, et al. Radiation carcinogenesis. Carcinogenesis. 2000;21(3):397-404.

6. Mc Laughlin PD, et al. Minimization of Radiation Exposure due to Computed Tomography in Inflammatory Bowel Disease ISRN Gastroenterol. 2012;2012:790-279.

7. OSullivan J, et al. An assessment of medical students' awareness of radiation exposures associated with diagnostic imaging investigations. Insights Imaging. 2010 May;1(2): 86-92.

8. Shiralkar $S$, et al. Doctors' knowledge of radiation exposure: questionnaire study. BJ. 2003 Aug 16;327(7411):371-2.

9. Wong CS, et al. A questionnaire study assessing local physicians, radiologists and interns' knowledge and practice pertaining to radiation exposure related to radiological imaging. Eur J Radiol. 2012 Mar;81(3):e264-8.
10. Keijzers GB, Britton CJ. Doctors' knowledge of patient radiation exposure from diagnostic imaging requested in the emergency department. Med J Aust. 2010;193(8): 450-453.

11. Jacob K1, Vivian G, Steel JR. X-ray dose training: are we exposed to enough? Clin Radiol. 2004 Oct;59(10):928-34.

12. Singh RK, et al. Teaching medical students about radiation protection - what do they need to know? Clin Radiol. 2008 Dec;63(12):1344-9.

13. Hagi SH, Medical student's knowledge of ionizing radiation and radiation protection. Saudi Med J. 2011;32(5): 179-83.

Correspondence address: Katarzyna Jończyk-Potoczna Zakład Radiologii Pediatrycznej 27/33 Szpitalna Street, 60-572 Poznań, Poland phone: +48 618491597 fax: +48618483362 email: sekrtg@skp.ump.edu.pl 